\title{
Do False Beliefs Predict Increased Circumcision Satisfaction in Men?
}

\author{
Stephen Moreton \\ Circ Facts, Warrington, England, U.K. \\ Email: stephenmoreton@totalise.co.uk
}

How to cite this paper: Moreton, S. (2020) Do False Beliefs Predict Increased Circumcision Satisfaction in Men? Advances in Sexual Medicine, 10, 41-55.

https://doi.org/10.4236/asm.2020.102003

Received: March 19, 2020

Accepted: April 14, 2020

Published: April 17, 2020

Copyright $\odot 2020$ by author(s) and Scientific Research Publishing Inc. This work is licensed under the Creative Commons Attribution International License (CC BY 4.0).

http://creativecommons.org/licenses/by/4.0/

\begin{abstract}
Medical male circumcision has been adopted by the WHO, and other professional public health and medical bodies, as a vital weapon in the fight against HIV. This has prompted a large body of research into the acceptability of the procedure, attitudes to it, and barriers to it. A systematic review of these studies found that satisfaction with circumcision is strongly associated with having accurate knowledge about it. A survey-based paper by Earp, Sardi and Jellison entitled "False beliefs predict increased circumcision satisfaction in a sample of US American men" is the only one to find the opposite. It therefore merits scrutiny. The present article presents the results of a critical examination of the study. Serious flaws were discovered. Half of the small number of 10 "true/false" statements used in its survey are questionable. All the large body of literature that contradicts the findings of Earp and co-workers is ignored. Importantly, the crucial question about whether dissatisfied circumcised males hold false beliefs about circumcision is not considered. Unlike most of the research on the acceptability of circumcision, the study is not motivated by a desire to evaluate the likely effectiveness of a public health measure, or how best to implement it. Rather it appears to be an attempt by a prominent anti-circumcision activist and his associates to generate a body of literature that they can then cite to further their cause. Considering this, and the serious flaws it contains, the study should be dismissed as misleading, biased and undermining public health.
\end{abstract}

\section{Keywords}

Circumcision, Satisfaction, Sexual Experience, Bodily Integrity, False Beliefs

\section{Introduction}

In a survey-based article entitled "False beliefs predict increased circumcision satisfaction in a sample of US American men", Earp, Sardi \& Jellison (hereafter 
ES\&J) [1] suggest that circumcised men who are satisfied with their status are satisfied because they hold false beliefs about male circumcision (MC). Presumably, if they held what ES\&J consider "true" beliefs, those men would be less satisfied. The present article provides a much-needed critical evaluation of the survey, including the questions asked, interpretation of the data obtained, the veracity of statements made by the authors, and a review of the literature.

\section{Evaluation}

\subsection{Overview}

ES\&J gave a questionnaire to an unstated number of workers at Amazon Mechanical Turk-https://www.mturk.com/-a crowdsourcing marketplace. Of those invited, 902 men completed the survey. These comprised 732 circumcised and 170 non-circumcised men. It was the authors' hope that this would be a representative sample. Besides asking the subjects to declare their circumcision status, they were quizzed about how satisfied they were with it on a scale of 1 to 5 , in order to arrive at a "Circumcision Satisfaction Score" that was then analyzed statistically. They were also given a list of ten statements about MC, five "true" and five "false", and asked to identify which were true and which were false. These are reproduced here in Table 1.

Table 1. The ten-item "Penile Anatomy and Circumcision Quiz" used by ES\&J and [in brackets] the answer that ES\&J deemed to be correct.

According to the American Academy of Pediatrics, there is good evidence that

1 being circumcised is associated with a lower incidence of urinary tract infections

[TRUE] (UTIs) in boys under the age of 2 .

If a baby boy is not circumcised shortly after birth, he will most likely require a circumcision anyway to correct medical problems before he turns 18 .

The percentage of men who are circumcised in the United States is significantly

3 greater than the percentage of men who are circumcised in most other English-speaking countries.

4 The foreskin is typically the least sensitive part of the penis to light touch.

After birth, a boy who has not been circumcised should have his foreskin "retracted" or pulled back as soon as possible to facilitate cleaning.

Some forms of non-therapeutic (ritual) female genital cutting that are described

6 by the World Health Organization (WHO) as "mutilation" are less physically invasive than male circumcision as it is typically performed in the United States.

Most medical associations around the world that have issued statements on

7 routine newborn male circumcision have concluded that the foreskin does not have any functions.

In the United States, during the late 19th and early 20th centuries, circumcision was advocated by mainstream doctors as a preventative measure against-or a "cure" for-masturbation.

According to most authoritative sources, approximately 100 circumcisions would

9 be needed to prevent 1 urinary tract infection (UTI) among boys with normally developing anatomy. routine newborn male circumcision recommend the procedure. 
According to the survey's results the circumcised men were (non-significantly) more satisfied with their status than the non-circumcised men. Taken at face value this contradicts the anti-circumcision campaigners' narrative that $\mathrm{MC}$ causes sexual dysfunction and psychological harm. In short, most circumcised men were happy about being circumcised, and perhaps were happier with their status than their non-circumcised peers.

Instead of accepting the findings at face value, ES\&J argued that those happily circumcised men held mistaken beliefs about MC and were more likely to get the true/false statements wrong, pointing to a statistically significant correlation between satisfaction and holding false beliefs. So, throwing away Occam's razor, the authors concluded the reason those circumcised men were satisfied was not the obvious one-i.e., that there is no detrimental consequence of being circumcised, and in fact being circumcised might even be better than not being circumcised-but rather that those men lacked what the authors considered "sufficient or accurate information regarding the anatomy and functions of the intact penis". They compared those supposedly "happily mistaken" men to women who had undergone female genital mutilation (FGM) but were satisfied with having undergone this procedure. This is a poor analogy as some forms of FGM are minor, as acknowledged in ES\&J's (true) statement number 6: "Some forms of non-therapeutic (ritual) female genital cutting that are described by the World Health Organization $(W H O)$ as 'mutilation' are less physically invasive than male circumcision as it is typically performed in the United States."

\subsection{Up to Half of the True/False Statements Are Problematic}

Troublingly, some of the true/false statements are irrelevant, ambiguous or incorrect.

Their statement number 4 reads: "The foreskin is typically the least sensitive part of the penis to light touch. [FALSE]". Indeed, the statement is false, but it is also irrelevant. It is erogenous sensation that matters, and this resides mostly in the glans and shaft, not the foreskin [2] [3]. Because it is irrelevant, it does not matter if some subjects get it wrong. It has no relevance to satisfaction.

Their statement number 7 reads: "Most medical associations around the world that have issued statements on routine newborn male circumcision have concluded that the foreskin does not have any functions. [FALSE]". This is a half-truth, and misleading. It is debated if the foreskin has any function at all, it may simply be an evolutionary relic, like the eyes of blind cave animals. But few (if any) medical associations say the foreskin has any important functions. Even the Royal Dutch Medical Association's strongly anti-circumcision statement fails to cite any credible evidence to this effect [4]. All this body could come up with was a study in which a man tried to copulate with a hole cut in a styrofoam cup, and a (questionable) observation that foreskin restoration is popular (argumentum ad populum).

The Royal Australasian College of Physicians' 2010 policy statement [5] has 
only a single paragraph on "Functions of the foreskin" in which they claim just two "functions": protection for the glans, and sensory function, but provide only one reference in support of the latter assertion, and none to support the former. The RCAP do not claim these functions are important, and they point to studies showing that removal of the foreskin has no adverse effect, thus implying that they do not consider these two "functions" to be important.

The Canadian Pediatric Society's 2015 position statement is similarly brief on foreskin "function" [6] and, like the RCAP, points to studies indicating no adverse effect from MC.

The most comprehensive evidence-based pediatric position statement is that of the American Academy of Pediatrics in 2012 [7] which found no evidence for any foreskin "function" and concluded that, "The literature review does not support the belief that male circumcision adversely affects penile sexual function or sensitivity, or sexual satisfaction, regardless of how these factors are defined."

The South African National Department of Health's lengthy guidelines [8] do not mention foreskin functions. Nor do those of the WHO [9]. Without wishing to labor the point, medical associations generally avoid detailed discussion of the topic of foreskin function. This does not mean that the foreskin has, or does not have, functions beyond that of skin elsewhere on the body, but it does imply that any putative "functions" are not considered to be important. ES\&J attribute too much significance to the limited commentary by medical associations regarding supposed foreskin functions. Furthermore, one could reword the statement thus: "Most medical associations around the world that have issued statements on routine newborn male circumcision have concluded that the foreskin does have functions. [FALSE]", which is the converse of what ES\&J say, yet still has a "false" answer. When a statement, and its reverse, both have the same "false" answer, the statement is too ambiguous to be of any use.

Significantly, all systematic reviews, including two meta-analyses have concluded that MC has no adverse effect on sexual function, sensation or satisfaction [10] [11] [12] [13]. Three of these are by non-partisans in the debate, working in non-circumcising cultures (China and Denmark, although the first author of the latter was Jewish). This is to be expected if the foreskin "does not have any functions".

Their statement number 8: "In the United States, during the late 19th and early 20 th centuries, circumcision was advocated by mainstream doctors as a preventative measure against-or a 'curé for-masturbation. [TRUE]" is largely false. MC only became popular in the U.S. following World War I, when it was observed that in the unhygienic conditions of the trenches some men developed foreskin-related medical problems. The "circumcision to stop masturbation" notion never got beyond a few quacks on the fringes, most famously John Harvey Kellogg. It was never a mainstream belief. This myth, popular amongst MC opponents, has been investigated and debunked [14].

Their statement number 9: "According to most authoritative sources, approximately 100 circumcisions would be needed to prevent 1 urinary tract infection 
(UTI) among boys with normally developing anatomy. [TRUE]" is arguably false. The 1 in 100 figure applies only to males during infancy, whereas over the lifetime UTI risk in non-circumcised males is 1 in 3 with a number needed to treat (NNT) of 4.3 [15]. If by "authoritative sources" one means primary, peer-reviewed, scientific ones, then only one primary source has reported the figure cited (for infant boys) [16]. Other studies found a NNT (for infant boys) of 83 [17] and 37 [18]. A higher figure of 195 has also been reported [19]. So only one of four "authoritative sources", not "most", support ES\&J's figure. In fairness to ES\&J two of these studies may not have been published when they wrote their manuscript. In any case, a layperson may not appreciate that even a NNT of 100 compares favorably with the NNT of some vaccines, a point made in comparison with infant influenza vaccine (NNT > 1000) [18]. Asking laypeople to assess a statistical statement that requires some technical understanding to appreciate is unreasonable.

Their statement number 10: "Most medical associations around the world that have issued statements on routine newborn male circumcision recommend the procedure. [FALSE]" is an oversimplification. It is recommended by the Centers for Disease Control and Prevention (CDC) in high-HIV settings [20], presumably with the support of local medical associations. Outside of those settings, statements range from saying infant MC is worthwhile and should be available, but stopping short of saying it should be routine [7] [21], to statements discouraging it but stopping short of seeking to ban it [4] [5]. Expecting lay people to be aware of these nuances is unfair and, in any case, whether they know about them or not is unlikely to influence their satisfaction with their own circumcision status.

Thus, fully half of the "true/false" statements used in the survey by ES\&J are problematic, even incorrect. To be useful they must be correct, unambiguous, and not requiring specialist expertise to properly understand. But the problems go deeper. Aside from the small number of statements (only 10), and their inaccuracy, it is difficult to escape the perception that the statements have been chosen to suit the first author's longstanding anti-circumcision agenda. Ultimately, all that their study demonstrates is that if one selects a small number of the "right" true/false statements one can "prove" a predetermined conclusion.

\subsection{Demographic Comparison of Groups}

Their Appendix contains a Supplementary Table of demographic parameters for the circumcised and the non-circumcised men in the study. However, a statistical comparison of the data for each group to show whether the groups were matched does not appear. Such a comparison, with $\mathrm{p}$ values, would be expected in any good epidemiological study. Curiously, some of their supposedly Jewish and Muslim men were reported as not being circumcised. In the footnote to the Table, they cite a 2005 study on prevalence of male circumcision in the USA, while ignoring the most recent data (in 2013) which was published by the US CDC [22], albeit for males aged 14 - 69 years, showing an overall rate of $80.4 \%$ (whites $90.8 \%$, blacks 75.7\% and Mexican-Americans 44.0\%). 


\subsection{Relevant Literature Ignored: A Systematic Review}

There is a substantial body of literature on the acceptability of MC, beliefs and attitudes regarding it, and barriers to it, a detailed understanding of these issues being essential to the WHO-backed MC program in high-HIV settings. Some of these studies investigated the relationship between the subjects' knowledge of MC, and their attitudes to it, which is also the subject of ES\&J's study. Astonishingly, their article makes no mention of this. Could this be because it is a consistent feature of these studies that when properly educated about MC, the majority of men are favorably inclined towards the procedure, whether they themselves are circumcised or not, and even larger numbers would have a son circumcised?

As this is of great relevance, and ES\&J's omission is a grave shortcoming, it merits particular attention. Accordingly, to assess the existing research on the topic a literature search was conducted. Criteria were:

1) Studies of men's attitudes towards $M C$ and the relationship between this and their knowledge of it.

2) Published in a peer-reviewed scientific journal or by a professional body.

3) English language.

4) Include data on circumcised males.

Studies were sorted into two categories. "Relevant" if they met the first three criteria above, and "Very relevant" if they included data on circumcised men. This latter point matters as, while ES\&J included both circumcised and non-circumcised males, their emphasis was on the former, for which their results were said to be statistically significant.

PubMed was searched using keywords "male", "circumcision", "attitudes", "satisfaction", "acceptable" and "education", while excluding "female", in various permutations which gave up to 390 hits. The Cochrane database was searched using "circumcision" and found an additional 15 items. The author's personal library, built up from 7 years of regular PubMed alerts using the single keyword "circumcision", yielded 155 papers relating to acceptability of MC, barriers to it, knowledge of it, and attitudes towards it. Searching the bibliographies of the relevant papers found a further 15. Figure 1 shows a PRISMA flowchart that summarizes the search strategy and results. 30 articles categorized as "relevant" were identified. While some of these included circumcised males in their samples, they did not separate data for these males sufficiently to merit inclusion in the "very relevant" category. The latter category comprised 12 articles, which are summarized in Table 2.

Because many of the 30 "relevant" papers have already been included in two systematic reviews [23] [24], they are not discussed individually here. Instead the reader is referred to those reviews, and the references therein, for details. A universal finding of these studies was that when non-circumcised men are well-informed about MC, particularly in regard to its health and hygiene benefits (for example in relation to HIV) large numbers (often majorities) express positive attitudes towards MC, even a desire to become circumcised. Large numbers of participants stated that they would have a son circumcised. 


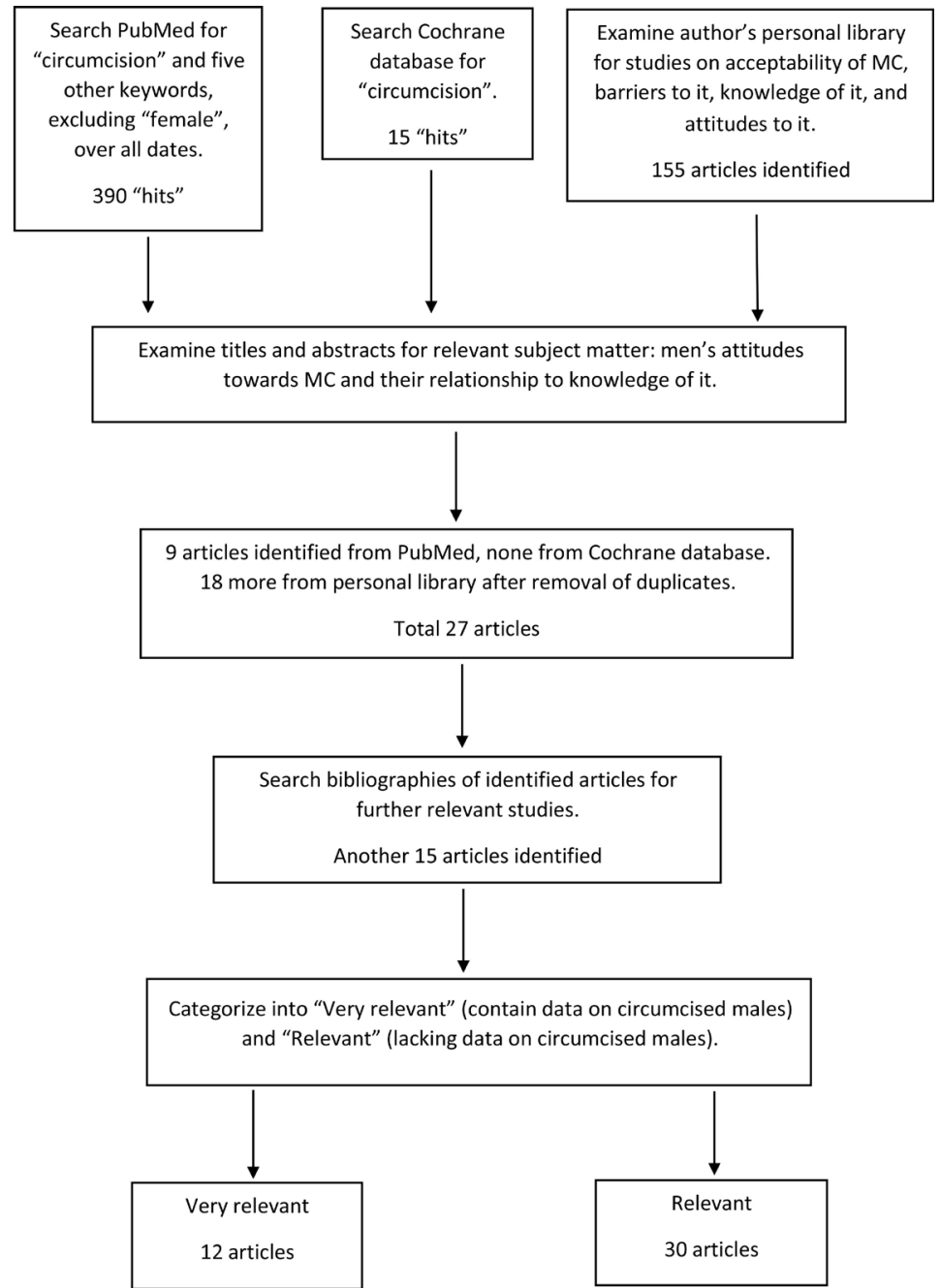

Figure 1. Search strategy diagram.

Table 2. Summary of "very relevant" studies identified in the literature search.

\begin{tabular}{llllc}
\hline Authors & Year & Country & Study type & Reference number \\
\hline Bengo et al. & 2010 & Malawi & Quantitative, cross-sectional & {$[30]$} \\
Carrasco et al. & 2019 & Various African & Systematic review & {$[23]$} \\
Jiang et al. & 2015 & China & Quantitative, cross-sectional & {$[38]$} \\
Kebaabetswe et al. & 2003 & Botswana & Quantitative, cross-sectional & {$[31]$} \\
Keetile \& Buwelo & 2016 & Botswana & Quantitative, cross-sectional & {$[32]$} \\
Lagarde et al. & 2003 & S. Africa & Quantitative, cross-sectional & {$[33]$} \\
Mavhu et al. & 2011 & Zimbabwe & Quantitative, cross-sectional & {$[35]$} \\
Mndzebel \& Tegegn & 2014 & Botswana & Quantitative, cross-sectional & {$[36]$} \\
Walcott et al. & 2013 & Jamaica & Quantitative, cross-sectional & {$[34]$} \\
Westercamp \& Bailey & 2007 & Various African & Systematic review & {$[24]$} \\
Yan et al. & 2015 & China & Quantitative, cross-sectional & {$[28]$} \\
Zamawe \& Kusamula & 2015 & Malawi & Mixed methods & {$[37]$} \\
\hline
\end{tabular}


The finding of an association between accurate knowledge about $\mathrm{MC}$ and favoring it, even desiring it, is consistent across different study types. Thus, when men's attitudes are compared before and after being given an educational session about MC, they invariably show an increase in willingness to be circumcised after the educational session. Likewise, when men's attitudes to MC are compared with their existing knowledge of the procedure, a positive attitude or desire to be circumcised correlates strongly with having accurate knowledge of MC. The information given to the participants, or knowledge on which they were assessed, mostly relates to the health and hygiene benefits of $\mathrm{MC}$, the effects on sexual function and the risks and limitations, as established in the scientific literature and taught by professional bodies involved in fighting the HIV epidemic. It may, therefore, be regarded as accurate. The studies also employed far more extensive questionnaires than the mere ten statements in the questionnaire used by ES\&J.

The aforementioned reviews only cover Africa. In a series of Chinese studies, the same effect was consistently observed [25] [26] [27] [28] [29]. After being educated about the risks and benefits of MC, and its effects on sexual function, majorities of men changed their minds and became positive about the procedure, or about having a son circumcised. Some even went on to get circumcised themselves. One of these studies [26] employed a questionnaire with 67 items, as opposed to the 10 used by ES\&J.

In short, for non-circumcised males, it is consistently observed that better knowledge of MC is associated with more favorable attitudes about MC, even a desire to be circumcised. Or to put it another way, the more they knew, the less satisfied with their own status they became. That millions of such men in high-HIV settings have gone on to be circumcised after receiving accurate information from professional health bodies indicates that these men, at least, became so dissatisfied with their non-circumcised status that they wanted to be rid of their foreskin. This contradicts ES\&J's finding that for non-circumcised males, dissatisfaction was (non-significantly) associated with holding what ES\&J considered to be "false beliefs".

Turning to circumcised males, the 12 articles identified here (Table 2) comprised the two systematic reviews, and 10 primary (mostly cross-sectional) studies. As with non-circumcised men, in all cases approval of MC increased with improved knowledge of the subject, in contradiction to ES\&J's finding. If anything, the effect may even be stronger in circumcised males. It was consistently found that circumcised males are more willing to have a son circumcised, than are non-circumcised ones, even when MC is not part of their culture [28] [30] [31] [32] [33] [34]. In some cases, more than $90 \%$ of circumcised men expressed willingness to circumcise a son [30] [31] [32] [34]. It is unlikely that men dissatisfied with their circumcised status would want to circumcise a son. These men are clearly satisfied, in addition to being well-informed.

It was also noted that circumcised men were better informed about MC than non-circumcised men [35] [36] [37] and "circumcised men were significantly 
more likely than non-circumcised men to agree about the advantages of circumcision" [33]. This is likely due to exposure to the African MC campaigns. Many men who had undergone MC considered that their sexual performance was enhanced [33] although in one study this could represent confounding due to their circumcisions largely stemming from foreskin problems [38]. In this latter study, on learning of the health benefits in relation to HIV most of these circumcised males were willing to promote MC in high-HIV settings. It may therefore be inferred that these men were satisfied with being circumcised. They would be unlikely to promote circumcision otherwise.

While satisfaction with being circumcised can be inferred, as explained above, it was the norm in the two studies that directly asked their participants about satisfaction. $51 \%$ were positive, $14 \%$ neutral, $35 \%$ negative in Malawi [37], $90 \%$ were satisfied, $10 \%$ indifferent in Botswana [31]. The reasons for the negativity in the Malawi study were not stated, but may relate to the procedure itself (pain, healing time, inconvenience, abstinence from sexual relations during the healing period) rather than to the final outcome.

From the above discussion, it is evident that most circumcised males in the studies were satisfied with their status, were well-informed about the procedure (more so than their non-circumcised peers), and their approval of the procedure increased with increasing knowledge of MC. As with the non-circumcised males, this is in direct contradiction to the finding of ES\&J. An objection may be made that ES\&J's study was of U.S. men circumcised neonatally, and the studies identified here were not. But it is hard to see why this should matter. The observations were consistent across diverse countries and cultures, and two countries (China and Jamaica) are outside the region in which MC is currently being promoted for reducing HIV infections. Some men in these studies had been circumcised neonatally [28], or in childhood [28] [30]. In others, although the age is not stated, neonatal or childhood MC is likely for many of the participants [34] [38].

ES\&J's study is anomalous. It is the only one to have come to the contrary conclusion. Its failure to mention any of the considerable body of opposing literature is striking and demands an explanation. Selectively ignoring all studies opposing ES\&J's anti-MC agenda can be regarded as either very sloppy scholarship, or overt bias.

\subsection{Biased Bibliography}

ES\&J's bibliography consists of 102 references. Of these 15 are authored, or co-authored, by Earp. Yet previously Earp has criticized an opponent for self-citation [39]. Strictly speaking, criticism for self-citing is ad hominem, as all it shows is that the author has written copiously on a topic, it says nothing about the quality of those writings, although in the case of ES\&J's paper it might imply a double standard on the part of the first author Earp, or at least a lack of self-awareness.

More tellingly, Earp's 15 self-citations are joined by a further 23 citations to other MC opponents, some of whom (Boyle, Darby, Frisch, Goldman, Hammond, 
Svoboda) are highly prominent in the anti-circumcision movement, and have histories of publishing low quality papers on the topic, often attracting harsh criticism [40]. Thus 38 of the citations (37\% of ES\&J's total) are to authors biased against MC. Only 3 papers that they cite (by first authors Kigozi, Mazor and Morris) are supportive of MC.

\subsection{Only Half the Question Was Addressed}

ES\&J asked if there was an association between false beliefs and MC satisfaction. One could reasonably ask the converse. Some men are dissatisfied with MC. Is this because these men hold false beliefs concerning it? As Earp has himself drawn attention to the existence of these unhappy circumcised men [41], it is surprising he has not included data on these men in the ES\&J study. A sample of such men could readily be obtained from anti-circumcision organizations, with which Earp is well-connected. But then it might be embarrassing if it transpired that dissatisfaction with MC was associated with false beliefs spread by those same organizations. A study of these unhappy circumcised men might utilise the true/false statements shown in Table 3, with some emphasis on "accurate information regarding the anatomy and functions of the intact penis" (to quote ES\&J).

Every one of the false statements in Table 3 is promulgated as "true" by anticircumcision groups, and every one of the true ones is either denied or downplayed by these MC opponents, as an Internet search will show. Will ES\&J take up this suggestion and see if dissatisfaction with circumcision is associated with incorrect answers to these statements?

Table 3. A list of true/false statements suitable for a survey of men unhappy with their circumcision status.

1 There are around 20,000 nerve endings in the foreskin.

[FALSE]

Approximately 117 babies die in the U.S. every year from circumcision complications.

[FALSE]

All medical and health organizations dealing with the global HIV epidemic

3 recognize that medical male circumcision is very effective at reducing female to male HIV transmission.

4 The foreskin has at least 16 known functions.

5 Infant, but not adult, circumcision is very effective at preventing penile cancer.

All the best quality scientific studies indicate that medical circumcision has no adverse effect on sexual function, sensation or satisfaction.

It has been proven that circumcision causes the surface of the glans to thicken and keratinize.

Nearly all men circumcised as adults, and thus able to compare before and after, are satisfied with the outcome.

9 Circumcision has no medical benefits. 


\section{Conclusions}

Most circumcised men are happy with their MC status and, as the literature review here shows, the more they know about MC the more positive they are about it. Conversely, the better educated that non-circumcised males are about MC the less satisfied they are with their own status, often to the point of wishing to become circumcised, or to have a son circumcised. In the U.S., those unhappy with being circumcised tend to be ones done as infants (and thus lacking before/after experience) [42]. Poor sexual function is also linked to dissatisfaction with being circumcised [42]. Could it be that being duped by anti-circumcision propaganda leads to distress, and hence sexual disfunction? MC opponents make many false claims about the effect of circumcision on sexual function and pleasure which may lead to psychological distress in those deceived [40]. This makes misleading articles such as ES\&J's particularly pernicious as they further a false narrative of circumcision causing harm.

This critical evaluation of "False beliefs predict increased circumcision satisfaction in a sample of US American men" leads to the conclusion that its findings contradict all other research in this area, are untrustworthy because of methodological flaws, and stem from overt bias by the study authors, which is evident throughout their article. Theirs' is one of a growing number of, often survey-based, works written by anti-MC campaigners to further their cause. Examination of these invariably finds them to be methodologically flawed, with highly selective bibliographies, citations that do not support the claims, and speculations passed off as fact. A recent, and particularly egregious, example being a survey, by noted anti-MC campaigners, of men who thought that their neonatal circumcision had harmed them [43], but which was shown to be scientifically worthless in a detailed and lengthy critique [44]. Given Earp's long history of writing anti-circumcision polemics, one can only suspect that the article critiqued here is just another attempt by circumcision opponents to build a body of literature that they can cite in support of their misguided agenda. They appear to feel the need to do this because most of the literature does not support their claims [40]. The need for journal editors and reviewers to be alert to the contents of manuscripts with a clear anti-circumcision agenda is becoming more important than ever, even more so considering MC's vital importance in HIV epidemic settings.

\section{Author's Affiliation}

Stephen Moreton Ph.D. is an editor of, and contributor to, CircFacts (http://www.circfacts.org), a website that critically examines arguments opposing male circumcision.

\section{Conflicts of Interest}

The author declares no conflicts of interest regarding the publication of this paper. 


\section{References}

[1] Earp, B.D., Sardi, L.M. and Jellison, W.A. (2018) False Beliefs Predict Increased Circumcision Satisfaction in a Sample of US American Men. Culture Health \& Sexuality, 20, 945-959. https://doi.org/10.1080/13691058.2017.1400104

[2] Cox, G., Krieger, J.N. and Morris, B.J. (2015) Histological Correlates of Penile Sexual Sensation: Does Circumcision Make a Difference? Sexual Medicine, 3, 76-85. https://doi.org/10.1002/sm2.67

[3] Schober, J.M., Meyer-Bahlberg, H.F.L. and Dolezal, C. (2009) Self-Ratings of Genital Anatomy, Sexual Sensitivity and Function in Men Using the "Self-Assessment of Genital Anatomy and Sexual Function, Male” Questionnaire. BJU International, 103, 1096-1103. https://doi.org/10.1111/j.1464-410X.2008.08166.x

[4] Royal Dutch Medical Association (KNMG) (2010) The Non-Therapeutic Circumcision of Male Minors. https://www.knmg.nl/circumcision

[5] Royal Australasian College of Physicians Division of Paediatrics and Child Health (2010) Circumcision of Infant Males.

https://www.racp.edu.au/docs/default-source/advocacy-library/circumcision-of-infa nt-males.pdf

[6] Sorokan, S.T., Finlay, J.T. and Jefferies, A.L. (2015) Newborn Male Circumcision. Paediatrics \& Child Health, 20, 311-320. https://doi.org/10.1093/pch/20.6.311

[7] American Academy of Pediatrics Task Force on Circumcision (2012) Male Circumcision. Pediatrics, 130, e756-e785. https://doi.org/10.1542/peds.2012-1990

[8] Department of Health Republic of South Africa (2016) South African National Guidelines for Medical Male Circumcision.

https://aidsfree.usaid.gov/sites/default/files/sa_mmc_guidelines.pdf

[9] World Health Organization (2009) Manual for Male Circumcision under Local Anaesthesia.

https://www.who.int/hiv/pub/malecircumcision/who_mc_local_anaesthesia.pdf

[10] Morris, B.J. and Krieger, J.N. (2013) Does Male Circumcision Affect Sexual Function, Sensitivity, or Satisfaction? - A Systematic Review. Journal of Sexual Medicine, 10, 2644-2657. https://doi.org/10.1111/jsm.12293

[11] Tian, Y., Liu, W., Wang, J-Z., Wazir, R., Yue, X. and Wang, K.-J. (2013) Effects of Circumcision on Male Sexual Functions: A Systematic Review and Meta-Analysis. Asian Journal of Andrology, 15, 662-666. https://doi.org/10.1038/aja.2013.47

[12] Shabanzadeh, D.M., Düring, S. and Frimodt-Møller, C. (2016) Male Circumcision Does Not Result in Inferior Perceived Male Sexual Function-A Systematic Review. Danish Medical Journal, 63, A5245.

https://ugeskriftet.dk/dmj/male-circumcision-does-not-result-inferior-perceived-m ale-sexual-function-systematic-review

[13] Yang, Y., Wang, X., Bai, Y. and Han, P. (2018) Circumcision Does Not Have Effect on Premature Ejaculation: A Systematic Review and Meta-Analysis. Andrologia, 50. https://doi.org/10.1111/and.12851

[14] Circumcision Choice (2017) Kellogg's Crusade. Blog Post. https://www.circumcisionchoice.com/single-post/Kellogg

[15] Morris, B.J. and Wiswell, T.E. (2013) Circumcision and Life-Time Risk of Urinary Tract Infection: A Systematic Review and Meta-Analysis. Journal of Urology, 189, 2118-2124. https://doi.org/10.1016/j.juro.2012.11.114

[16] Christakis, D.A., Harvey, E., Zerr, D.M., Feudtner, C., Wright, J.A. and Connell, 
F.A. (2000) A Trade-Off Analysis of Routine Newborn Circumcision. Pediatrics, 105, 246-249. https://pediatrics.aappublications.org/content/105/Supplement_2/246

[17] Ellison, J.S., Dy, G.W., Fu, B.J., Holt, S.K., Gore, J.L. and Merguerian, P.A. (2018) Neonatal Circumcision and Urinary Tract Infections in Infants with Hydronephrosis. Pediatrics, 142, e20173703. https://doi.org/10.1542/peds.2017-3703

[18] Eisenberg, M.L., Galusha, D., Kennedy, W.A. and Cullen, M.R. (2018) The Relationship between Neonatal Circumcision, Urinary Tract Infection, and Health. World Journal of Men's Health, 36, 176-182. https://doi.org/10.5534/wjmh.180006

[19] To, T., Agha, M., Dick, P.T. and Feldman, W. (1998) Cohort Study on Circumcision of Newborn Boys and Subsequent Risk of Urinary-Tract Infection. The Lancet, 352, 1813-1816. https://doi.org/10.1016/S0140-6736(98)02392-7

[20] Hines, J.Z., Onkemetse, C.N., Malaba, K., Zegeye, T., Serrem, K., Odoyo-June, E., Nyrienda, R.K., Msungama, W., Nkanaunena, K., Come, J., Canda, M., Nhaguiombe, H., Shihepo, E.K., Zemburuka, B.L.T., Mutandi, G., Yoboka, E., Mbayiha, A.H., Maringa, H., Bere, A., Lawrence, J.J., Lija, G.J.I., Simbeye, D., Kazaura, K.. Mwiru, R.S., Talisuna, S.A., Lubwama, J., Kabuye, G., Zulu, J.E., Chituwo, O., Mumba, M., Xaba, S., Mandisarisa, J., Baack, B.N., Hinkle, L., Grund, J.M., Davis, S.M. and Toledo, C. (2017) Scale-Up of Voluntary Medical Male Circumcision Services for HIV Prevention-12 Countries in Southern and Eastern Africa, 2013-2016. CDC Morbidity and Mortality Weekly Report, 66, 1286-1290.

https://doi.org/10.15585/mmwr.mm6647a2

[21] CDC (2018) Information for Providers to Share with Male Patients and Parents Regarding Male Circumcision and the Prevention of HIV Infection, Sexually Transmitted Infections, and Other Health Outcomes.

https://stacks.cdc.gov/view/cdc/58456

[22] Introcaso, C.E., Xu, F., Kilmarx, P.H., Zaidi, A. and Markowitz, L.E. (2013) Prevalence of Circumcision among Men and Boys Aged 14 to 59 Years in the United States, National Health and Nutrition Examination Surveys 2005-2010. Sexually Transmitted Diseases, 40, 521-525. https://doi.org/10.1097/01.OLQ.0000430797.56499.0d

[23] Carrasco, M.A., Wilkinson, J., Kasdan, B. and Fleming, P. (2019) Systematic Review of Barriers and Facilitators to Voluntary Medical Male Circumcision in Priority Countries and Programmatic Implications for Service Uptake. Global Public Health, 14, 91-111. https://doi.org/10.1080/17441692.2018.1465108

[24] Westercamp, N. and Bailey, R.C. (2007) Acceptability of Male Circumcision for Prevention of HIV/AIDS in Sub-Saharan Africa: A Review. AIDS and Behavior, 11, 341-355. https://doi.org/10.1007/s10461-006-9169-4

[25] Huang, J., Jian, J., Yang, X., Lian, B., Qin, B., Upur, H., Zhong, C., Wang, Q., Wang, Q., Ruan, Y., Deng, W., Xie, P., Liao, Y., Xu, N., Zou, Y., Wei, F., Ye, L. and Liang, H. (2016) Assessment of Different Intervention Models of Male Circumcision and Their Preliminary Effectiveness in Reducing HIV Incidence among Drug Users in Western China. AIDS Research and Human Retroviruses, 32, 972-980. https://doi.org/10.1089/aid.2016.0019

[26] Jiang, J., Huang, J., Yang, X., Ye, L., Wei, B., Deng, W., Weu, S., Qin, B., Upur, H., Zhong, C., Wang, Q., Wang, Q., Ruan, Y., Wei, F., Xu, N., Xie, P. and Liang, H. (2013) Acceptance of Male Circumcision among Male Rural-to-Urban Migrants in Western China. AIDS Research and Human Retroviruses, 29, 1582-1588. https://doi.org/10.1089/aid.2013.0156

[27] Yang, X., Abdullah, A.S., Wei, B., Juang, J., Deng, W., Qin, B., Yan, W., Wang, Q., Zhong, C., Wang, Q., Ruan, Y., Zou, Y., Xie, P., Wei, F., Xu, N. and Liang, H. (2012) 
Factors Influencing Chinese Male's Willingness to Undergo Circumcision: A Cross-Sectional Study in Western China. PLoS ONE, 7, e30198. https://doi.org/10.1371/journal.pone.0030198

[28] Yan, W.-L., Wang, C.-C., Huang, Y.-D., Yimiti, D., Wang, Q. and Upur, H. (2015) Parental Factors Affecting the Circumcision of Non-Muslim Chinese Boys Include Education and Family History. Acta Paediatrica, 104, e569-e576.

https://doi.org/10.1111/apa.13142

[29] Zhou, B., Ning, C., McCann, C.D., Liao, Y., Yang, X., Zou, Y., Jiang, J., Liang, B., Abdullah, A.S., Qin, B., Upur, H., Zhong, C., Ye, L. and Liang, H. (2017) Impact of Educational Interventions on Acceptance and Uptake of Male Circumcision in the General Population of Western China: A Multicenter Cohort Study. Scientific Reports, 7, Article No. 14931. https://doi.org/10.1038/s41598-017-13995-9

[30] Bengo, J.M., Chalulu, K., Chinkhumba, J., Kazembe, L., Maleta, K.M., Masiye, F. and Mathanga, D. (2010) Situation Analysis of Male Circumcision in Malawi. Draft Report by College of Medicine. https://www.malecircumcision.org/sites/default/files/document_library/Malawi_Ma le_Circumcision_Situation_Analysis_Report_2010-04-21.pdf

[31] Kebaabetswe, P., Lockman, S., Mogwe, S., Mandevu, R., Thior, I., Essex, M. and Shapiro, R.L. (2003) Male Circumcision: An Acceptable Strategy for HIV Prevention in Botswana. Sexually Transmitted Infections, 79, 214-219.

https://doi.org/10.1136/sti.79.3.214

[32] Keetile, M. and Buwelo, M. (2016) Factors Associated with Acceptability of Child Circumcision in Botswana-A Cross Sectional Survey. BMC Public Health, 16, 1053. https://doi.org/10.1186/s12889-016-3722-5

[33] Lagarde, E., Dirk, T., Puren, A., Rain-Taljaard, R. and Auvert, B. (2003) Acceptability of Male Circumcision as a Tool for Preventing HIV Infection in a Highly Infected Community in South Africa. AIDS, 17, 89-95. https://doi.org/10.1097/00002030-200301030-00012

[34] Walcott, M.M., Jolly, P.E., Ehiri, J.E., Funkhauser, E., Kempf, M.C., Hickman, D., Aung, M. and Zhang, K. (2013) Factors Associated with the Acceptability of Male Circumcision among Men in Jamaica. PLoS ONE, 8, e75074.

https://doi.org/10.1371/journal.pone.0075074

[35] Mavhu, W., Buzdugan, R., Langhaug, L.F., Hatzold, K., Benedikt, C., Sherman, J., Laver, S.M., Mundida, O., Woelk, G. and Cowan, F.M. (2011) Prevalence and Factors Associated with Knowledge of and Willingness for Male Circumcision in Rural Zimbabwe. Tropical Medicine \& International Health, 16, 589-597. https://doi.org/10.1111/j.1365-3156.2011.02744.x

[36] Mndzebel, S.L. and Tegegn, G.A. (2014) Knowledge, Attitude and Acceptance of Voluntary Male Medical Circumcision among Male Students Attending Botswana University. Journal of Public Health and Epidemiology, 7, 6-14. https://doi.org/10.5897/JPHE2014.0671

[37] Zamawe, C.O.F. and Kusamula, F. (2015) What Are the Social and Individual Factors That Are Associated with Undergoing Male Circumcision as an HIV Prevention Strategy? A Mixed Methods Study in Malawi. International Health, 8, 170-178. https://doi.org/10.1093/inthealth/ihv061

[38] Jiang, J., Su, J., Yang, X., Huang M., Deng, W., Huang, J., Liang, B., Qin, B., Upur, H., Zhong, C., Wang, Q., Wang, Q., Ruan, Y., Ye, L. and Liang, H. (2015) Acceptability of Male Circumcision among College Students in Medical Universities in Western China: A Cross-Sectional Study. PLoS ONE, 10, e0135706.

https://doi.org/10.1371/journal.pone.0135706 
[39] Earp, B.D. and Darby, R. (2015) Does Science Support Infant Circumcision? The Skeptic, 25, 23-30.

https://www.skeptic.org.uk/society-culture/the-first-cut/does-science-support-infan t-circumcision/

[40] Morris, B.J., Moreton, S. and Krieger, J.N. (2019) Critical Evaluation of Arguments Opposing Male Circumcision: A Systematic Review. Journal of Evidence Based Medicine, 12, 263-290. https://doi.org/10.1111/jebm.12361

[41] Earp, B.D. (2015) Sex and Circumcision. American Journal of Bioethics, 15, 43-45. https://doi.org/10.1080/15265161.2014.991000

[42] Bossio, J.A. and Puckall, C.F. (2018) Attitude toward One's Circumcision Status Is More Important than Actual Circumcision Status for Men's Body Image and Sexual Functioning. Archives of Sexual Behavior, 47, 771-781.

https://doi.org/10.1007/s10508-017-1064-8

[43] Hammond, T. and Carmack, A. (2017) Long-Term Adverse Outcomes from Neonatal Circumcision Reported in a Survey of 1,008 Men: An Overview of Health and Human Rights Implications. International Journal of Human Rights, 21, 189-218. https://doi.org/10.1080/13642987.2016.1260007

[44] Bailis, S.A., Moreton, S. and Morris, B.J. (2019) Critical Evaluation of a Survey Claiming "Long-Term Adverse Outcomes from Neonatal Circumcision". Advances in Sexual Medicine, 9, 67-109. https://doi.org/10.4236/asm.2019.94006 